\title{
The molecular archaeology of a mitochondrial death effector: AIF in Drosophila
}

\author{
N Joza ${ }^{1,2,3,4}, K_{\text {Galindo }}{ }^{5}$, JA Pospisilik ${ }^{1}$, P Benit ${ }^{6}$, M Rangachari $^{1}$, EE Kanitz ${ }^{1}$, Y Nakashima ${ }^{1}$, GG Neely ${ }^{1}$, P Rustin ${ }^{6}$, JM Abrams ${ }^{\star, 5}$, \\ $\mathrm{G} \mathrm{Kroemer}^{\star, 2,3,4}$ and JM Penninger ${ }^{\star, 1}$
}

Apoptosis-inducing factor (AIF) is a phylogenetically conserved redox-active flavoprotein that contributes to cell death and oxidative phosphorylation in Saccharomyces cerevisiae, Caenorhabditis elegans, mouse and humans. AlF has been characterized as a caspase-independent death effector that is activated by its translocation from mitochondria to the cytosol and nucleus. Here, we report the molecular characterization of AIF in Drosophila melanogaster, a species in which most cell deaths occur in a caspase-dependent manner. Interestingly, knockout of zygotic D. melanogaster AIF (DmAIF) expression using gene targeting resulted in decreased embryonic cell death and the persistence of differentiated neuronal cells at late embryonic stages. Although knockout embryos hatch, they undergo growth arrest at early larval stages, accompanied by mitochondrial respiratory dysfunction. Transgenic expression of DmAIF misdirected to the extramitochondrial compartment ( $\Delta \mathrm{N}-\mathrm{DmAIF})$, but not wild-type DmAIF, triggered ectopic caspase activation and cell death. $\Delta \mathrm{N}$-DmAIF-induced death was not blocked by removal of caspase activator Dark or transgenic expression of baculoviral caspase inhibitor p35, but was partially inhibited by Diap1 overexpression. Knockdown studies revealed that $\Delta \mathrm{N}$-DmAIF interacts genetically with the redox protein thioredoxin-2. In conclusion, we show that Drosophila AIF is a mitochondrial effector of cell death that plays roles in developmentally regulated cell death and normal mitochondrial function.

Cell Death and Differentiation (2008) 15, 1009-1018; doi:10.1038/cdd.2008.24; published online 29 February 2008

Apoptosis-inducing factor (AIF) was originally characterized as a phylogenetically conserved cell death mediator, a mitochondrial protein that translocates to the nucleus following an apoptotic stimulus and triggers chromatin condensation and DNA degradation. ${ }^{1}$ In the nucleus, AIF binds to DNA and recruits an endonuclease complex, which includes cyclophilin $A,{ }^{2}$ to trigger large-scale $(\sim 10 \mathrm{~kb})$ fragmentation of DNA. More recently, AIF has been implicated as a cell survival factor required for the maintenance of mitochondrial respiratory complex function and/or for scavenging reactive oxygen species. In particular, the Harlequin $(H q)$ mouse strain, which carries a hypomorphic mutation in AIF, develops late-onset neuronal and retinal degeneration accompanied by increased neuronal expression of oxidative stress markers. ${ }^{3}$ Other groups have found that inactivation of AIF by gene targeting in mice or RNA interference in cell lines results in decreased protein expression and function of respiratory complex I, and leads to impaired cell growth and survival. ${ }^{4-8}$ Thus, AIF has multiple functions, both in cell death execution and in mitochondrial energy homeostasis.

The notion that AIF is an important cell death effector is supported by several loss-of-function studies. In $\mathrm{Hq}$ mice, glutamate-induced excitotoxic cell death of hippocampal neurons is attenuated compared to wild-type controls. ${ }^{9} \mathrm{Hq}$ mice also display smaller infarct volumes after cerebral hypoxia-ischemia, ${ }^{10,11}$ and $\mathrm{Hq}$ neuronal cortical cells exhibit partial resistance to cell death in response to serum deprivation and PARP-1 signaling.,12 In Caenorhabditis elegans, knockdown of the AIF ortholog WAH-1 results in a reduction in developmental cell death; ${ }^{13}$ in Saccharomyces cerevisiae, AIF null mutants exhibit decreased death in response to oxidative stress and chronological aging. ${ }^{14}$ These findings support the idea of a phylogenetic conservation of AIF-mediated cell death. Importantly, AIF has been shown to exert its cytotoxic effects in a caspase-independent manner. ${ }^{15,16}$

\footnotetext{
${ }^{1}$ IMBA, Institute of Molecular Biotechnology of the Austrian Academy of Sciences, Dr. Bohrgasse 3, 1030 Vienna, Austria; ${ }^{2}$ INSERM, U848, F-94805 Villejuif, France; ${ }^{3}$ University Paris-Sud, Paris 11, F-94805 Villejuif, France; ${ }^{4}$ Institut Gustave Roussy, 39 rue C Desmoulins, F-94805 Villejuif, France; ${ }^{5}$ Department of Cell Biology, University of Texas Southwestern Medical Center, 5323 Harry Hines Boulevard, Dallas, TX 75390, USA and 'INSERM, U676, University 'Denis Diderot' Hopital, Robert Debré, 75019 Paris, France

${ }^{*}$ Corresponding author: JM Penninger, IMBA, Institute of Molecular Biotechnology GmbH, Dr. Bohrgasse 3, 1030 Vienna, Austria. Tel: + 431 79044; Fax: + 43179044 4701; E-mail: Josef.penninger@imba.oeaw.ac.at or

G Kroemer, INSERM, U848, Institut Gustave Roussy, F-94805 Villejuif, France. Tel: + 3314211 60 46; Fax: + 331421160 47; E-mail: kroemer@igr.fr or JM Abrams, UT Southwestern Medical Center, 5323 Harry Hines Blvd, Dallas, TX 75390-9039, USA. Tel: + 1214648 9226; Fax: + 1214 648 8694; E-mail: john.abrams@utsouthwestern.edu

Keywords: mitochondria; oxidative phosphorylation; Drosophila

Abbreviations: AEL, after egg laying; AIF, apoptosis-inducing factor; AO, acridine orange; Apaf-1, apoptotic protease-activating factor-1; Cyt $c$, cytochrome $C$; Diap, Drosophila inhibitor of apoptosis; DIG, digoxigenin; DmAIF, D. melanogaster apoptosis-inducing factor; G3PDH, glycerol-3-phosphate dehydrogenase; $\mathrm{Hq}$, Harlequin; mAIF, mouse AIF; MLS, mitochondrial localization signal; NLS, nuclear localization sequence; PCD, programmed cell death; UV, ultraviolet; VNC, ventral nerve cord

Received 26.4.07; revised 14.1.08; accepted 15.1.08; Edited by E Baehrecke; published online 29.2.08
} 
There is an ongoing debate as to the contribution of mitochondria to the cell death program of different species. In mammalian cells, the 'point of no return' of the intrinsic cell death pathway occurs when the outer mitochondrial membrane permeabilizes and several apoptogenic proteins, including cytochrome $c($ Cyt $c)$, Smac/DIABLO, AIF and endonuclease $G$, are released from the mitochondrial intermembrane space. ${ }^{17}$ In Drosophila melanogaster, several reports have found no indication that Cyt $c$ is released from mitochondria during apoptosis ${ }^{18-21}$ and, until recently, there was little evidence for mitochondrial involvement in the fly cell death program. Two recent reports, however, showed that mitochondrial remodeling is an important process in the cell death program in Drosophila, and that Cyt $c$ translocates to the cytosol in Reaper- and Hid-induced, but not ultraviolet (UV)-induced, cell death. ${ }^{22,23}$ Moreover, Cyt $c$ is required for caspase activation associated with spermatid differentiation. $^{24}$ Thus, there is emerging evidence of a critical involvement of mitochondria in apoptosis in Drosophila, implying that cell death mechanisms between flies and mammals are more broadly similar than previously believed.

Genetic studies in flies have shown that most cell death that occurs during development and in response to chemotherapeutic agents can be blocked by genetic inhibition of caspases or activators of caspases. ${ }^{25-27}$ One interesting exception is the death of nurse cells during late oogenesis, in which caspase activity is not detectable and transgenic expression of caspase inhibitors p35 and Drosophila inhibitor of apoptosis (Diap) 1 has no effect on egg chamber development. ${ }^{28}$ Nevertheless, in light of the physiologically relevant cytotoxic functions of AIF orthologs in C. elegans and yeast, we asked whether an AIF ortholog in $D$. melanogaster similarly functions as a cell death effector.

\section{Results and Discussion}

Identification of the Drosophila AIF ortholog. The fly genome contains a single open reading frame (SD03428, CG7263) with $>65 \%$ similarity to mammalian AIF. The predicted D. melanogaster AIF (DmAIF) protein (674 amino acids, aa) exhibits $50 \%$ sequence identity and $68 \%$ similarity to mouse AIF (mAIF), and 33\% identity and 50\% similarity to $C$. elegans AIF (WAH-1) (Figure 1A). In silico analysis revealed a mitochondrial localization sequence (MLS, Supplementary Figure 1a). Expression of cDNA constructs corresponding to C-terminally tagged DmAIF, either full length or lacking the first 176 aa including the MLS ( $\triangle \mathrm{N}$-DmAIF), yielded proteins of the expected size (Supplementary Figure $1 \mathrm{~b}$ and $\mathrm{c}$ ). Subcellular localization of these proteins revealed a mitochondrial distribution for full-length DmAIF and a non-mitochondrial distribution for $\triangle \mathrm{N}$-DmAIF (Figure $4 \mathrm{~b}$ and Supplementary Figure 2a), confirming that the $\mathrm{N}$ terminus contains an MLS. Regions of mAIF critical for its oxidoreductase activity are highly conserved in DmAIF, particularly a putative FAD-binding domain (aa 183-323 and 462-539), an NADH-binding domain (aa 324-461), as well as the core consensus of the classical Rossman fold involved in direct binding of $\mathrm{NADH}$ and
FAD (aa 196-201 and 367-372) (Figure 1A). ${ }^{30,31}$ This may suggest that, like mammalian AIF, ${ }^{32}$ DmAIF is redox active.

Two other salient features of mAIF, a putative nuclear localization sequence (NLS) at aa 377-387 and 445-450 (red asterisks, see legend to Figure $1 \mathrm{~A}$ ) and residues required for binding to DNA (red circles), are absent in DmAIF (Figure 1A), ${ }^{1,29}$ suggesting that DmAIF might not translocate to the nucleus. A Myc-tagged full-length DmAIF transgene expressed under the control of the eyeless promoter yielded a cytoplasmic punctate staining that colocalized with mitochondrion-targeted GFP (Supplementary Figure 2a). However, exposure of these flies to $\gamma$-irradiation to trigger apoptosis did not result in mitochondrio-nuclear translocation of myc-tagged DmAIF (Supplementary Figure 2b). Similar results were obtained in the SL2 Drosophila cell line: overexpression of the lethal protein GRIM (Supplementary Figure 3a) or UVC irradiation (Supplementary Figure 3b) failed to trigger DmAIF redistribution from mitochondria. Together, these findings suggest that DmAIF remains sequestered in mitochondria, at least under the apoptotic conditions tested here and in contrast to mammalian AIF. 2,13,16

In mammalian tissues, AIF is ubiquitously expressed..$^{30}$ Similarly, DmAIF was found expressed throughout all stages of development but appeared downregulated at the pupal stage, as revealed by northern blotting and in situ hybridization of embryos (Figure 1B and C). In summary, we identified the Drosophila ortholog of AIF, which shares high sequence similarity, mitochondrial localization and an expression pattern similar to mammalian AIF.

Developmental and bioenergetic defects due to inactivation of the DmAIF gene. We generated DmAIFmutant flies by gene targeting, using 'ends-in' homologous recombination. ${ }^{33}$ The targeting construct consisted of the entire DmAIF genomic locus (Figure 2a); base pair insertions designed to disrupt the open reading frame of $D m A I F$ were introduced into exons 3 and 6 . One gene-targeted line, designated $D m A I F^{T 52}$, was validated by genomic Southern blotting using DNA probes flanking the $5^{\prime}$ and $3^{\prime}$ end of the DmAIF locus (Figure 2b). Sequencing of a PCR-cloned region of the targeted locus confirmed that the introduced point mutations were correctly targeted (data not shown). Western blotting of lysates from wild-type and mutant larvae showed absence of DmAIF protein in gene-targeted animals (Figure 2c); we thus designate this mutant line $D m A I F^{\mathrm{KO}}$.

Strikingly, $D m A / F^{\mathrm{KO}}$ larvae were markedly reduced in size, although their general anatomy (segmental pattern, development of the dorsal vessel, tracheal system and fat body) appeared normal, suggesting normal organogenesis coupled to deficient growth (Figure 2d). Zygotic homozygous $D m A I F^{K O}$ mutant embryos hatch, but larvae die within several days after egg laying (AEL) (Figure 2e). A time-course analysis of lethality showed that by day $6 \mathrm{AEL}$ approximately half of $D m A I F^{\mathrm{KO}}$ mutant larvae were dead; by day 8 no viable $D m A I F^{K O}$ mutants were recovered (Figure $2 \mathrm{e}$ ). Mutant larvae were noticeably smaller in size within hours after hatching (data not shown). By day 4, when most wild-type flies had reached the third-instar larval (L3) stage, the $D m A I F^{K O}$ mutant flies were at the first- and second-instar larval (L1 and L2) stages (Figure 2f). No third-instar larvae (L3) were recovered 

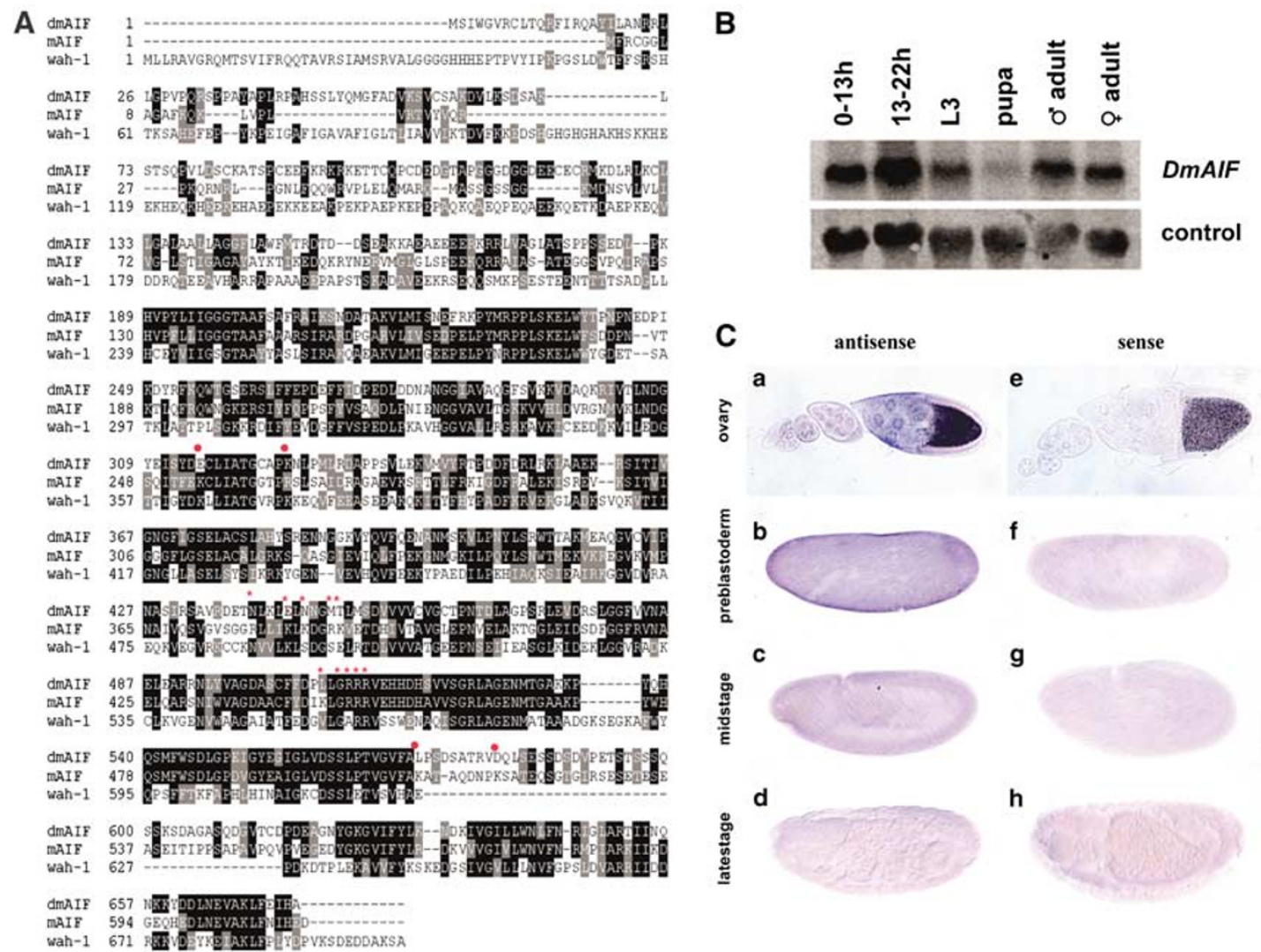

g
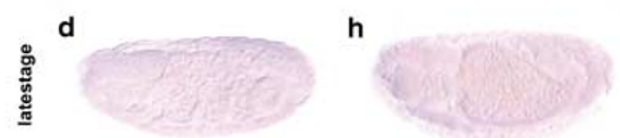

Figure 1 Cloning and analysis of expression of DmAIF. (A) Sequence alignment of DmAIF, mAIF and WAH-1. Black shading and gray shading indicate identical and similar amino-acid residues, respectively. Sequence alignment was performed using ClustalW and Boxshade programs using translations of SD03428 (DmAIF), AF100927 (mAIF) and AY147006 (WAH-1). Red asterisks represent the NLS identified in mAIF, ${ }^{1}$ and red circles indicate residues in mAlF required for binding DNA; ${ }^{29}$ both regions are poorly conserved in DmAIF. (B) Northern blot analysis of DmAIF expression during early (0-13 h) and late (13-22 h) embryogenesis, third-instar larvae (L3), pupae (P), and male $(\mathrm{M})$ and female $(\mathrm{F})$ adults. Expression was detected by northern blotting using a full-length DmAIF cDNA probe. (C) Whole-mount in situ analysis of DmAIF expression during embryogenesis. DmAIF antisense (a-d) and sense (e-h) cDNA probes labeled with DIG were used to detect DmAIF expression in wild-type embryos at various stages of development

even in the few surviving mutants at days 7 and 8 . Growth arrest at $\mathrm{L} 1$ of $D m A / F^{\mathrm{KO}}$ larvae was exacerbated in crowded culture conditions (Figure 2f), suggesting an underlying bioenergetic defect. Accordingly, we observed that the enzymatic activity of respiratory chain complexes I and IV from $D m A I F^{K O}$ larvae was strongly reduced as compared to heterozygous controls (Figure 2g). Moreover, the level of ATP was severely reduced in DmAIF ${ }^{\mathrm{KO}}$ larvae compared to controls (Figure 2h). In light of previous findings demonstrating that AIF is required for normal oxidative phosphorylation in human cell lines, mice and yeast, ${ }^{5-7}$ these data point to an evolutionarily conserved bioenergetic function for AIF.

Selective defects in developmentally regulated cell death in $D m A I F^{k O}$ embryos. We next analyzed developmental programmed cell death (PCD) in mid-stage embryos by acridine orange (AO) staining. DmAIF embryos had less incidence of developmental PCD (Figure $3 b$ and d) compared to wild-type (Figure $3 a$ and c) embryos (stage 12). As a consequence of the reduction of PCD, we investigated the pattern and persistence of extra cells in the central nervous system by different neuronal markers. Antibody staining against kruppel and $\mathrm{dHb} 9$ was performed. The pattern of neuronal cells appeared unperturbed; however, DmAI $F^{K O}$ embryos had extra cells in both the ventral nerve cord (VNC) and Bolwig's organ (Figure $3 f$ and i) compared to wild-type embryos (Figure $3 e$ and h). The number of VNC cells was comparable in abdominal region $\mathrm{A} 1$, but the presence of extra cells increased in a posterior manner in $D m A I F^{\mathrm{KO}}$ embryos from abdominal segments A2-A6 compared to wild-type embryos (Figure $3 \mathrm{~g}$ ). DmAIF ${ }^{\mathrm{KO}}$ embryos had on average more than three extra cells in the Bolwig's organ (Figure 3j). Since kruppel is a pan-neuronal CNS marker, we looked at a specific subset of neurons in the VNC. As anticipated, antibody staining against $\mathrm{dHb} 9$ revealed the presence of extra cells along the lateral edges of the VNC in DmAIF $F^{K O}$ embryos (Figure $3 \mathrm{l}$ and $\mathrm{m}$ ) compared to wild-type embryos (Figure 3k).

Cell death-associated organ disruption mediated by extramitochondrial DmAIF. We next generated transgenic flies carrying epitope-tagged UAS-DmAIF and UAS$\triangle N$-DmAIF constructs (Figure 4a). Control experiments, in which these constructs were transfected into SL2 cells, revealed that full-length DmAIF-myc co-stained with the 
a
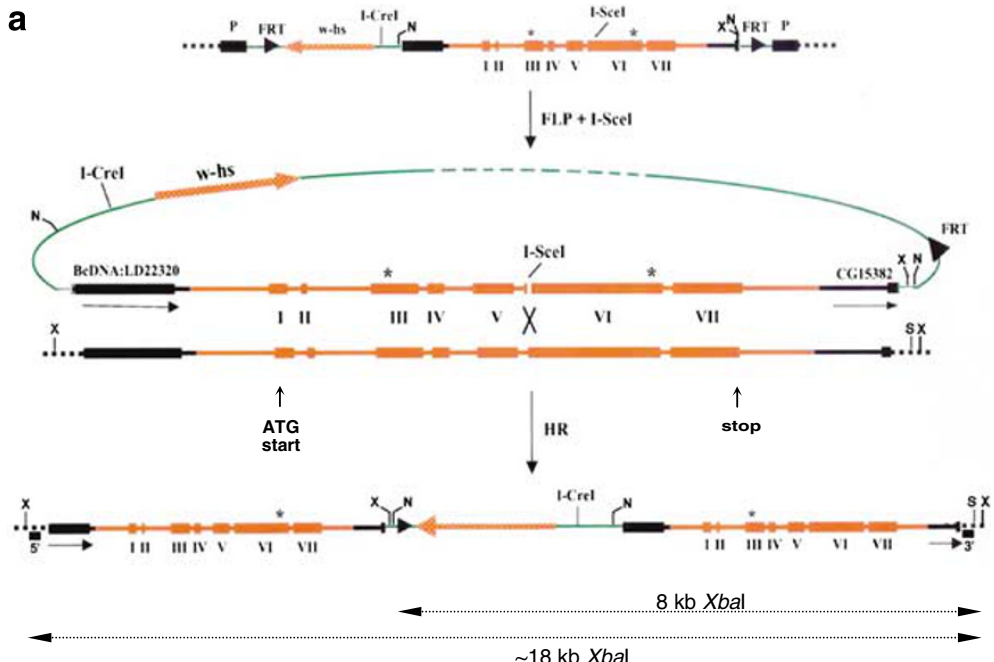
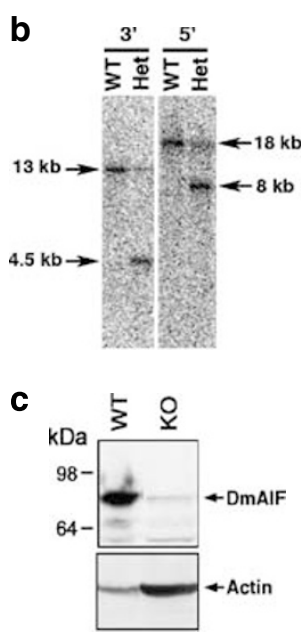

d

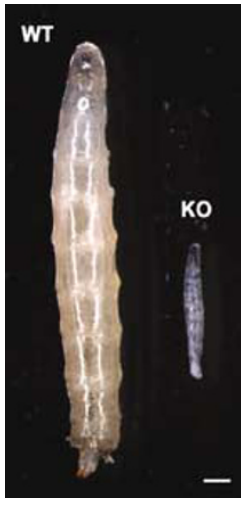

e

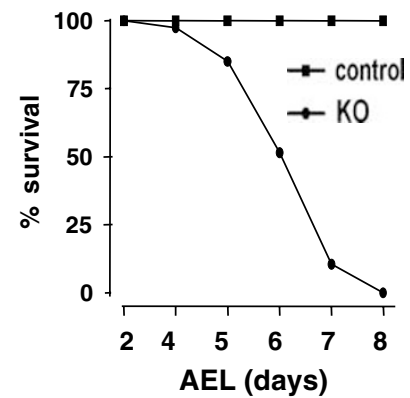

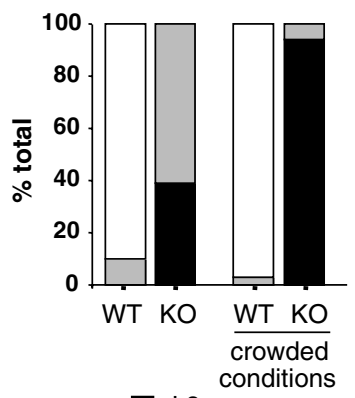

g

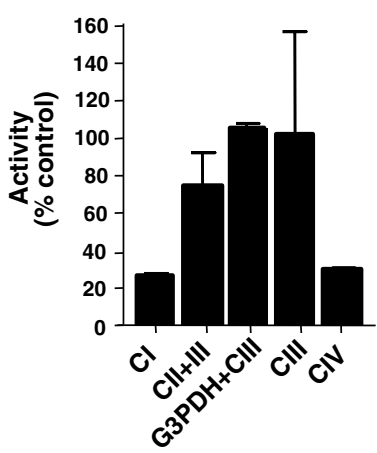

h

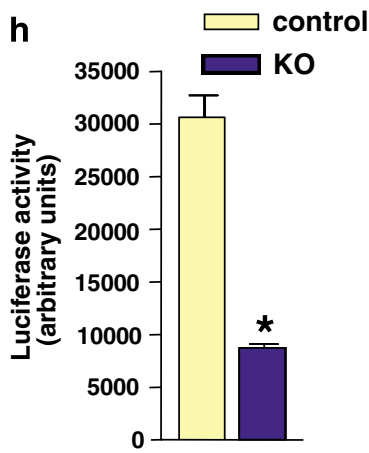

Figure 2 DmAIF gene-targeted flies exhibit growth arrest at early larval stage and defects in mitochondrial respiration. (a) Targeting scheme for the DmAIF locus. Illustrated are the donor construct, the native DmAIFlocus and the mutated locus after gene targeting. Boxes indicate exons. The donor construct consisted of a 5-kb fragment encompassing the entire DmAIF gene (in red) and carrying frameshifting base pair insertions (asterisks) within exons 3 and 6 . A germline transformant strain bearing this construct on the $\mathrm{X}$ chromosome was used for targeted mutagenesis. The resulting targeted lesion is a tandem duplication of two variants of the $D m A I F$ gene, each bearing the original introduced mutations as confirmed by sequencing of the endogenous locus. The predicted genomic fragment sizes after digestion with $X b a l$ for the native locus and mutated locus are illustrated. HR, homologous recombination; FRT, FLP recombination target; w-hs, $w^{\text {hs }}$ expression cassette; N, Notl; S, Sall; X, Xbal. (b) Southern blot of genomic DNA from wild-type (WT) and DmAIF $/$ + heterozygous (Het) flies. Genomic DNA was digested with Notl and Sall (left panel) or Xbal only (right panel) and analyzed by Southern blotting using $3^{\prime}$ and $5^{\prime}$ flanking probes, respectively, as indicated in panel a. (c) Lysates from wild-type (WT) and DmAIF $F^{K O}$ homozygous mutant (KO) first-instar larvae were immunoblotted with anti-DmAIF and anti-actin antibodies. (d) Gross morphology of wild-type (WT) and DmAIF ${ }^{\mathrm{KO}}$ homozygous mutant (KO) larvae on days 4 and 6 , respectively, after egg laying. Bar represents $300 \mu \mathrm{m}$. (e) Survival of heterozygous (control, squares) and DmAlF ${ }^{\mathrm{KO}}$ homozygous mutant (KO, circles) flies. AEL, after egg laying. (f) DmAlF $F^{\mathrm{KO}}$ mutants are arrested at the first- (L1) and second-instar (L2) larval stages. Four days after egg laying, larvae were staged as $\mathrm{L} 1-3$, based on mouth hook structure and size. $n>100$. In another experiment, larvae were grown at high density to mimic nutrient limitation, and staged on day 4 . (g) Selective defects in respiratory complex activities in DmAIF ${ }^{\mathrm{KO}}$ mutants. Mitochondria were isolated from first- and second-instar wild-type and DmAI ${ }^{\mathrm{KO}}$ mutants and assessed for respiratory complex activities. Values represent activities of each complex (I, II + III, G3PDH + III, III, IV) from mutant mitochondria as a percentage of control (100\%). Values are mean \pm S.E.M. for two independent experiments. Similar results were obtained in another experiment not assessing complex II activity using only early first-instar larvae. (h) ATP levels from second-instar (day $3 \mathrm{AEL}$ ) heterozygous and homozygous $D m A I F^{K O}$ mutants, determined by ATP-dependent luciferase activity normalized to protein content. Means \pm S.E.M. are shown from two experiments. ${ }^{*} P<0.05$

mitochondrion-selective cationic dye Mitotracker Red CMXRos, while $\Delta$ N-DmAIF-myc (encoding aa 177-674) no longer localized to mitochondria (Figure $4 \mathrm{~b}$ ). We next tested the function of DmAIF in vivo by generating transgenic flies expressing these constructs. While flies expressing fulllength DmAIF in the eye appeared normal (Figure 4c), flies expressing $\Delta \mathrm{N}$-DmAIF exhibited a marked reduction in eye size (Figure $4 \mathrm{c}$, right panels) and disorganized ommatidial architecture (Figure 4d). The eye phenotype exhibited 100\% penetrance but variable expressivity, ranging from a roughened appearance and normal size to a severe $(>80 \%)$ reduction in size. Eye discs from ey-GAL4;UAS$\triangle N$-DmAIF third-instar larvae stained positive for activated caspases (Figure 4e) and the vital dye AO (Supplementary Figure 1d), indicating that the eye phenotype was caused by ectopic cell death.

As AIF has been shown to trigger PCD in the absence of caspase function, ${ }^{16,34}$ we determined whether DmAIF is 

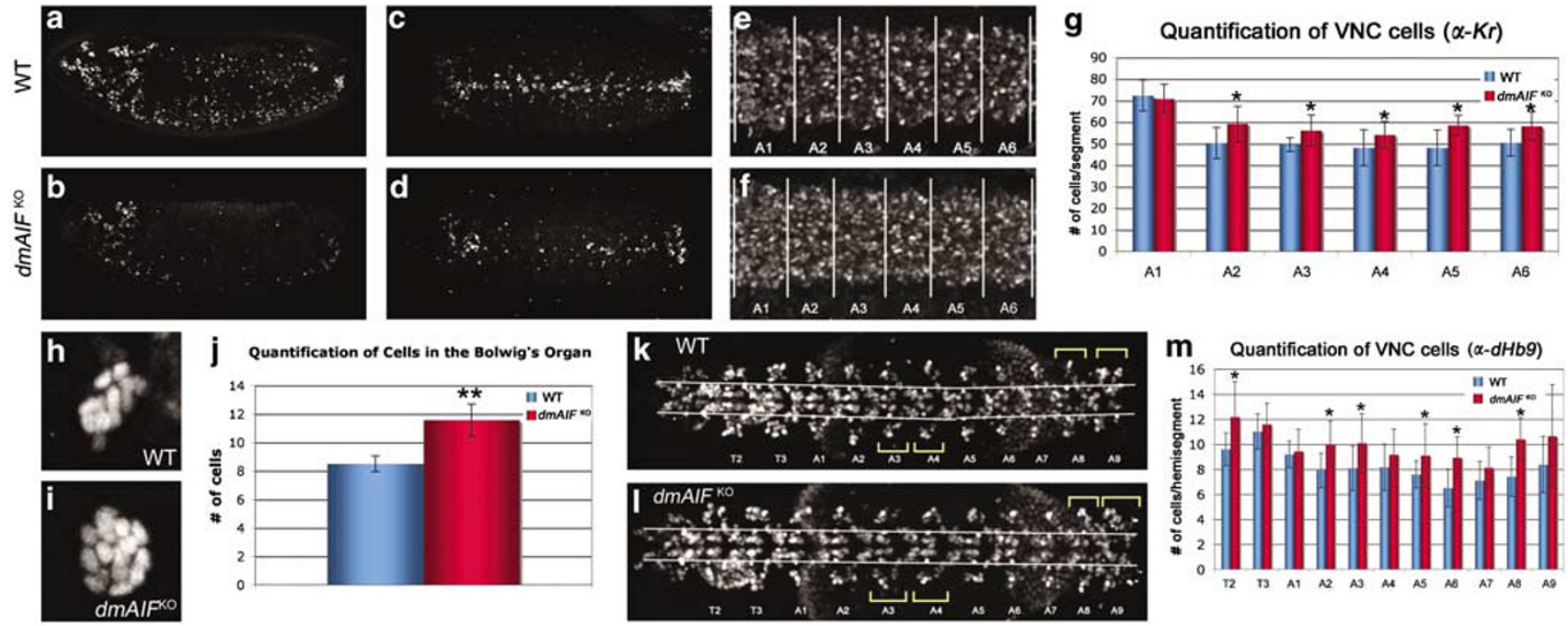

Figure $3 D m A I F^{K O}$ embryos exhibit extra cells in the central nervous system. (a-d) AO staining on wild-type (a, c) and $D m A I F^{K O}$ embryos (b, d). (e-g) DmAIF ${ }^{K O}$ embryos (f) show extra cells by $\alpha$-dHb9 antibody staining compared to wild-type embryos (e). (g) Histogram of extra cells in the VNC by $\alpha-K r$ antibody staining $(n=8)$. (h-j) $D m A l F^{K O}$ embryos (i) have extra cells in the Bolwig's organ compared to wild-type embryos $(\mathbf{h})(\alpha-K r)$. (j) Histogram of cell counts in the Bolwig's organ $(n=6)$. $(\mathbf{k}-\mathbf{m})$ DmAIF ${ }^{K 0}$ embryos (I) have extra cells in the VNC by $\alpha$-dHb9 antibody staining compared to wild-type embryos (k) (yellow brackets). (m) Histogram of extra VNC cells by $\alpha$-dHb9 antibody staining $(n=6)$. Error bars represent \pm S.D.; ${ }^{*} P<0.05 ;{ }^{* \star} P<0.005$, Student's $t$-test

a caspase-independent cell death promoter by crossing ey-GAL4;UAS- $\triangle N$-DmAIF to mutant lines genetically deficient in caspases or activators of caspases. Inactivation of the Drosophila Apaf-1 (apoptotic protease-activating factor-1)/ CED4 ortholog Dark, the fly apical caspase DREDD, Dmp53, or loss of one copy of the H99 locus (which spans the essential regulators grim, reaper and hid) failed to modulate the $\Delta \mathrm{N}$ DmAIF phenotype (Figure 5a, small panels). ${ }^{26,27}$ However, expression of the caspase inhibitors Diap1 or Diap2 did significantly, albeit incompletely, ameliorate the reduced eye size (Figure 5a). Interestingly, coexpression of the baculoviral caspase inhibitor p35 and $\Delta \mathrm{N}$-DmAIF in the eye failed to abolish the DmAIF-mediated disruption of normal eye architecture, and paradoxically, dramatically enhanced the phenotypic changes promoted by $\triangle \mathrm{N}$-DmAIF (Figure $5 \mathrm{a}$ ). Similar results were obtained when the $\Delta N-D m A I F$ transgene was targeted to dorsal and thoracic bristles using pnr-GAL4. In this case, expression of $\Delta N-D m A I F$ resulted in a reduction in bristle number and size, which could not be rescued by $\mathrm{p} 35$ coexpression; rather, p35 enhanced the phenotype (Figure 5b).

Altogether, these findings suggest that transgene-enforced expression of extramitochondrial DmAIF can kill cells and perturb tissue organization in a manner that may depend at least partially on caspase activity.

Thioredoxin-2 as an obligate mediator of extramitochondrial DmAIF cytotoxicity. To determine whether the cytotoxic effects of $\Delta \mathrm{N}-\mathrm{DmAIF}$ represent a specific, genetically modifiable cell death pathway, we set out to identify binding partners of DmAIF. A yeast two-hybrid screen of a mouse brain cDNA library was performed using mAIF as bait. One major hit (2 among 45 clones) was thioredoxin (txn1). The genome of $D$. melanogaster encodes several members of a thioredoxin family, including deadhead (dhd) and thioredoxin-2 (DmTrx-2). ${ }^{35}$ Since DmTrx-2-mutant flies were available in public stock centers, we chose to investigate in detail a potential interaction between DmTrx-2 and DmAIF. We transfected Flag-tagged DmTrx-2 into wild-type and AIF-deficient mouse embryonic stem cells, ${ }^{36}$ and confirmed that DmTrx-2 immunoprecipitates with endogenous AIF (Figure 6a). To test whether this physical interaction is important for the proapoptotic action of DmAIF in vivo, we expressed an inverted repeat of the DmTrx-2 gene (UAS-DmTrx-2-IR) to silence DmTrx-2 mRNA expression in flies expressing $\Delta \mathrm{N}$-DmAIF. Importantly, downmodulation of DmTrx-2 abolished the eye-disruptive effects of $\Delta \mathrm{N}$-DmAIF overexpression, and re-established normal eye morphology (Figure $6 b$ and $c$ ).

Among newly eclosed $F_{1}$ generation ey-GAL4 flies, we noticed a relative increase in numbers of those that carry both the UAS- $\triangle N-D m A I F$ and UAS-DmTrx-2-IR transgenes compared to UAS- $\triangle N-D m A I F$ alone. We had earlier observed that expression of $\Delta \mathrm{N}$-DmAIF in the eye results in lethality at the pre-eclosion stage (Figure 6d), likely due to the leaky tissue expression of $\triangle \mathrm{N}$-DmAIF under ey-GAL4 control. In contrast, in two independent lines of UAS-DmTrx-2-IR, downregulation of DmTrx-2 ameliorated the relative numbers of eclosing flies expressing $\Delta \mathrm{N}$-DmAIF, and almost restored expected Mendelian frequencies (Figure 6d). Thus, these data suggest that the redox protein DmTrx-2 cooperates with DmAIF to facilitate cell killing, and that DmAIF-induced cytotoxicity represents a specific and genetically modifiable cell death pathway.

\section{Conclusion}

There has been no previous report of the implications of AIF in Drosophila. In this paper, we demonstrate an evolutionarily conserved function of AIF in organismal growth and mitochondrial respiration. Loss of zygotic expression of DmAIF 


\begin{tabular}{|c|c|c|}
\hline \multirow[b]{2}{*}{ DmAIF-myc } & j & 674 \\
\hline & MLS & myc \\
\hline$\Delta \mathrm{N}-\mathrm{Dm}$ AIF-m & & myc \\
\hline
\end{tabular}

b

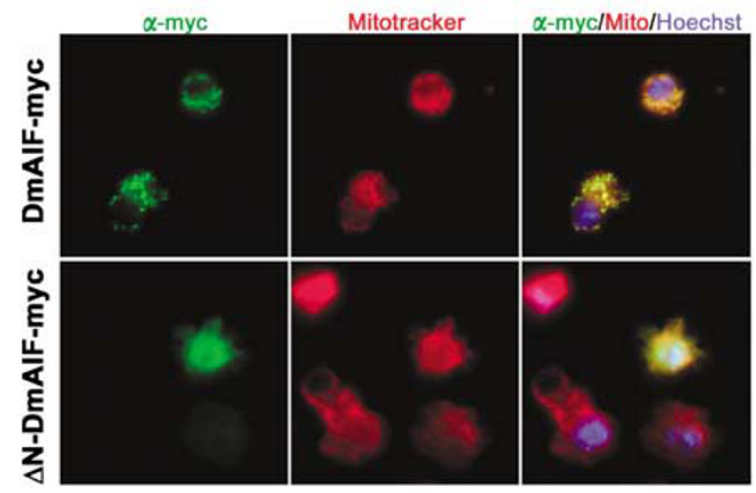

$\mathbf{C}$
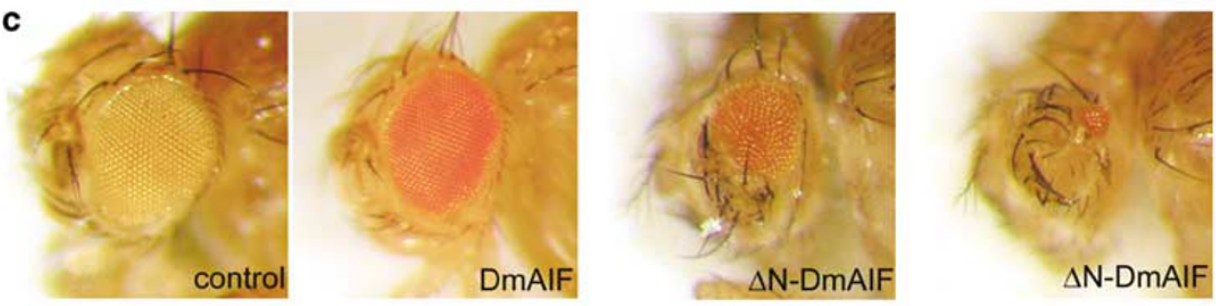

d

control

$\triangle \mathrm{N}$-DmAIF

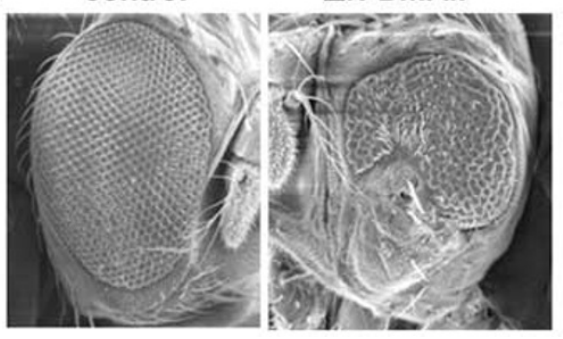

e

control

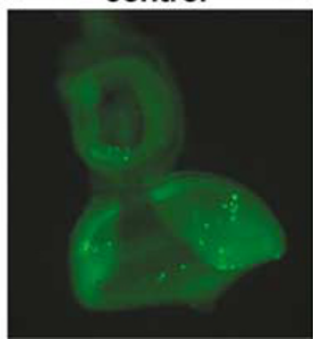

$\Delta \mathrm{N}-\mathrm{DmAIF}$

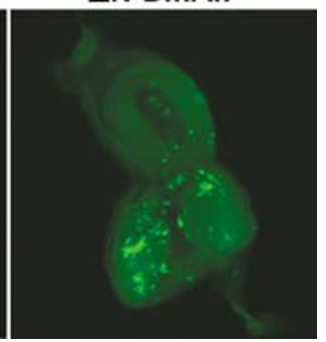

Figure 4 Misexpression of DmAIF triggers ectopic cell death. (a) Schematic diagram of Myc-tagged full-length DmAIF (DmAlF-myc) and an amino-terminal deletion mutant lacking amino acids 1-176, including the presumptive mitochondrial import sequence ( $\Delta \mathrm{N}$-DmAlF-myc). (b) Localization of DmAIF-myc and $\Delta \mathrm{N}$-DmAIF-myc in SL2 cells. Expression of constructs was detected using anti-c-Myc antibody (green). Cells were counterstained with Mitotracker Red CMXRos (red) and Hoechst 33342 (blue) to visualize mitochondria and nuclei, respectively. (c) Stereomicroscopic photographs of eyes from control and transgenic flies expressing full-length (DmAlF-myc) and $\mathrm{NH}_{2}$ terminally truncated $(\triangle \mathrm{N}-\mathrm{DmAIF}$ ) forms of DmAIF under the control of the eypromoter. Note that the left panel of $\triangle \mathrm{N}$-DmAIF is representative of the majority of flies expressing $\triangle \mathrm{N}$-DmAIF; the right panel of $\triangle \mathrm{N}$-DmAIF is typical of $\sim 5 \%$ of flies. Genotypes are as follows: ey-GAL4/CyO (control); ey-GAL4,UAS-DmAIF-myc/CyO (DmAIF); ey-GAL4,UAS- $\triangle N$-DmAIF/CyO ( $\triangle \mathrm{N}$-DmAIF). (d) Scanning electron micrographs of eyes from (left) control ey-GAL4/CyO and (right) ey-GAL4,UAS- $\triangle N$-DmAIF/CyO flies. (e) Immunostaining of eye discs from (left) control ey-GAL4/CyO and (right) ey-GAL4,UAS- $\triangle N-D m A I F / C y O$ third-instar larvae with an antibody recognizing cleaved caspase 3 (Asp175) and visualized using Alexa 488-conjugated secondary antibodies

results in arrested larval growth, loss of viability and defective functioning of the respiratory complexes, a phenotype analogous to mice carrying a null allele of Aif, which results in embryonic growth retardation and lethality by day 11.5 of gestation. ${ }^{5}$ Similarly, knockdown of the $C$. elegans AIF ortholog WAH-1 leads to a slower growth rate and smaller brood size, although the authors of the report did not investigate a potential mitochondrial bioenergetic defect. ${ }^{13}$ Our results, thus, confirm a role for AIF in maintaining energy homeostasis in the cell. However, the precise role of AIF in supporting normal respiratory complex function remains unknown.
Our findings also shed light on a proapoptotic function of AIF in Drosophila. Flies lacking zygotic DmAIF expression exhibit fewer dying cells during embryogenesis relative to wild-type flies and a modest increase in neuronal cells along the VNC. The persistence of extra cells occurred predominantly in the posterior abdominal segments of mutant embryos. The basis for this regional specificity is unclear but may involve redundant expression of AIF homologous genes that are known to exist in mammals yet have not been characterized in Drosophila. In any event, it will be interesting to investigate germline DmAIF mutant clones, as maternal expression of DmAIF protein is substantial (Figure 1C). 
a

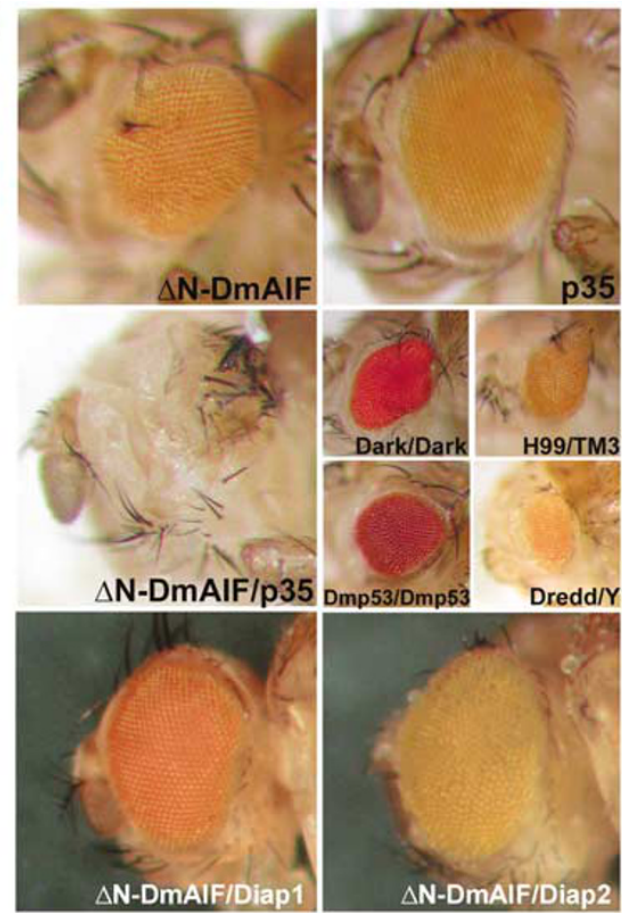

b

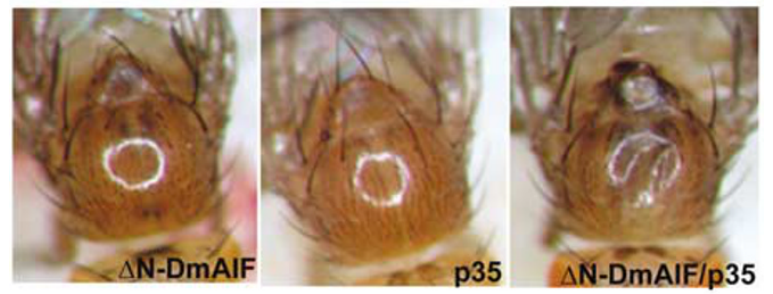

Figure 5 Epistasis analysis of DmAIF and several cell death regulators. (a) Large panels show flies expressing $\triangle \mathrm{N}-\mathrm{DmAlF}$, p35, or both transgenes under control of ey-GAL4, and flies coexpressing $\triangle \mathrm{N}$-DmAIF and Diap1, or $\triangle \mathrm{N}$-DmAIF and Diap2. The small panels show flies expressing $\Delta \mathrm{N}$-DmAIF in genetic backgrounds homozygous for Dark or Dmp53, hemizygous for Dredd (Dredd/Y) or heterozygous for $\mathrm{Df}(3 \mathrm{~L}) \mathrm{H} 99$ (H99/TM3). Note that female ey-GAL4,UAS- $\Delta N$ $D m A I F I+$ flies, which display a less severe eye phenotype than male flies (shown in Figure 4c), were used in these epistasis experiments. (b) Flies carrying the pnrGAL4 transgene were crossed to UAS- $\triangle N-D m A I F$, UAS-p35 or UAS- $\triangle N$ DmAIF;UAS-p35 flies. Note the exacerbated reduction in eye size (a) and loss of thoracic bristles (b) in flies coexpressing $\triangle \mathrm{N}$-DmAIF and $\mathrm{p35}$. Genotypes in large panels in (a) are ey-GAL4,UAS- $\triangle N$-DmAIFI + ( $\triangle N$-DmAIF); ey-GAL4,UAS-p35 (p35); ey-GAL4,UAS- $\triangle N$-DmAIFIUAS-p35 ( $\triangle N$-DmAIF;p35); ey-GAL4,UAS- $\Delta N$ DmAIF;UAS-Diap1 ( $\triangle \mathrm{N}$-DmAIF;Diap1); and ey-GAL4,UAS- $\triangle N$-DmAIF;UAS-Diap2 ( $\triangle \mathrm{N}$-DmAIF;Diap2). Genotypes in panel b are pnr-GAL4,UAS- $\triangle N-D m A I F /+(\Delta \mathrm{N}$ DmAIF); UAS-p35/+ ;pnr-GAL4/+ (p35); and UAS-p35/ + ;pnr-GAL4,UAS- $\Delta N$ $D m A I F /+(\Delta N-D m A I F ; p 35)$

Our finding of a proapoptotic function of Drosophila AIF in developmentally regulated cell death raises several interesting issues. Presumably, Drosophila AIF, like its counterparts in $C$. elegans and mammals, requires translocation to the nucleus to mediate cell killing, where its putative endonuclease and chromatin-condensing activities are activated. ${ }^{2,13}$ However, in two different systems in which apoptosis was induced (UV-irradiated SL2 cells and $\gamma$-irradiated eye disc cells), we did not observe release of DmAIF from mitochondria, suggesting that stress-induced apoptosis does not involve DmAIF. A number of studies have reported that Drosophila Cyt $c$ also remains localized within mitochondria in apoptotic cells, in apparent evolutionary contrast to the situation in higher organisms. ${ }^{18,20,21}$ On the other hand, it was recently reported that Cyt $c$ translocates to the cytosol in Reaper- and Hid-induced, but not UV-induced, cell death. ${ }^{22,23}$ Thus, it remains possible that, like Cyt $c$, DmAIF translocation from mitochondria depends on the apoptotic stimulus and cellular context. Furthermore, our results do not exclude a role for non-nuclear proapoptotic effects of DmAIF. Indeed, mammalian AIF can exert part of its biochemical effects by arresting protein translation, through a direct interaction with elF3g, ${ }^{37}$ and the $C$. elegans AIF ortholog WAH-1 can enhance phosphatidylserine exposure of dying cells through a direct interaction with a phospholipids scramblase. ${ }^{38}$ This means that, in different species, AIF does mediate major non-nuclear alterations in cellular physiology (protein synthesis arrest and loss of plasma membrane asymmetry) that are considered to be hallmarks of the apoptotic process, and it remains to be seen whether these changes are also influenced by DmAIF.

Our data provide some clues into the mechanisms through which AIF exerts its cytocidal effect. Overexpression of a truncated form of DmAIF lacking its N-terminal mitochondrialtargeting signal (but not flies expressing full-length DmAIF) triggered ectopic caspase activation and cell death. It is noteworthy that another GAL4 driver tested in combination with UAS- $\Delta N-D m A I F$, namely $g m r-G A L 4$, did not elicit obvious phenotypic changes ( $\mathrm{N}$ Joza and JM Penninger, unpublished data). This may reflect an intrinsic resistance of certain cell types to DmAIF-mediated death or insufficient expression of the transgene (i.e., DmAIF may require relatively high levels of expression to trigger cell death). In addition, we obtained somewhat conflicting results with regard to whether DmAIF requires caspases to mediate cell killing. While expression of $\mathrm{p} 35$ failed to suppress (and paradoxically, enhanced) DmAIF-mediated cell death, a significant inhibition was observed with expression of Diap1 or Diap2. Since p35 and Diap1 are reported to inhibit distinct caspases (in particular, p35 fails to inhibit $\mathrm{DRONC}^{39}$ ), it seems that a caspase may contribute to the cell death triggered by Drosophila AIF. Indeed, overexpression of the C. elegans AIF ortholog WAH-1 lacking a mitochondrial-targeting signal induces cell death that is partially blocked in a ced-3 (caspase)-mutant background. ${ }^{13}$ This contrasts with the caspase-independent cell death reported for AIF orthologs in S. cerevisiae and mammals. ${ }^{14,16}$ Thus, there may be a species-specific requirement for caspases in AIF-mediated cell killing.

Finally, we have identified a novel interaction between Drosophila AIF and the redox protein thioredoxin-2 (DmTrx2). Knockdown of DmTrx-2 suppressed DmAIF-induced cell death, suggesting that DmAIF cooperates with DmTrx-2 in execution of cell death. Future work will clarify whether this interaction is relevant to DmAIF-dependent developmental cell death in the fly embryo.

\section{Materials and Methods}

Cloning and characterization of DmAIF. A search of the BDGP EST database identified a sequence (SD03428) with significant homology to MAIF and 
b

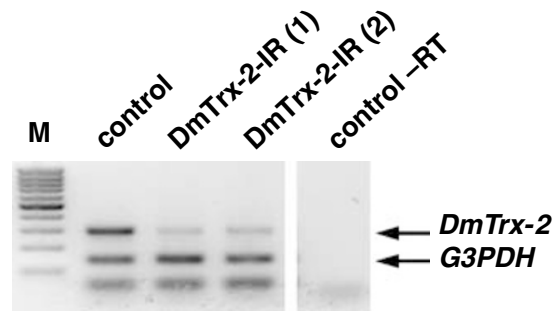

Cy-GAL4;UAS- $\triangle N-D m A I F$

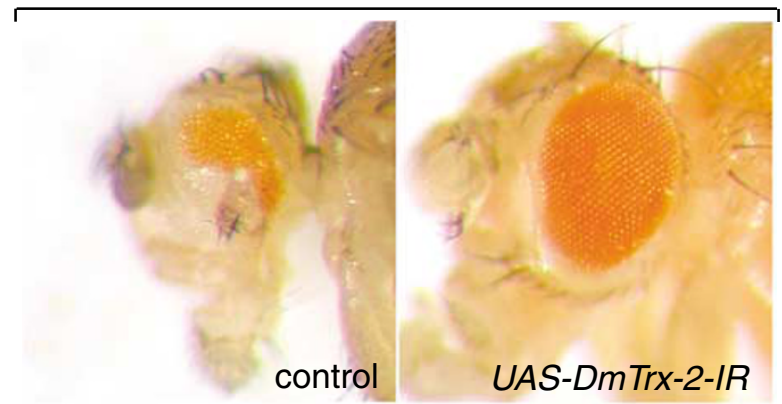

control

d
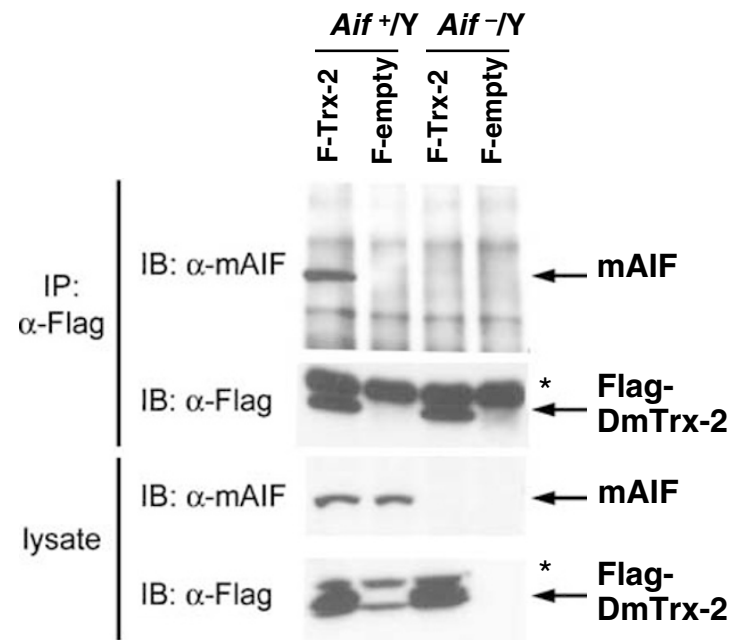

\begin{tabular}{|c|c|c|c|}
\hline \multirow{2}{*}{$\begin{array}{l}\text { Expected } \\
\text { Frequency }\end{array}$} & \multicolumn{2}{|c|}{$U A S-\triangle N-D m A I F$} & \multirow[b]{2}{*}{ - } \\
\hline & - & UAS-DmTrx-2-IR & \\
\hline $50 \%: 50 \%$ & $12 \%(35)$ & & $88 \%(258)$ \\
\hline $50 \%: 50 \%$ & & $45 \%(91)$ & $55 \%(110)$ \\
\hline $33 \%: 67 \%$ & & $28 \%(31)$ & $72 \%(81)$ \\
\hline
\end{tabular}

Figure 6 Drosophila thioredoxin-2 (DmTrx-2) modulates DmAlF-induced cell death. (a) Flag-tagged DmTrx-2 (Flag-DmTrx-2) was expressed in Aif ${ }^{+} / Y$ or Aif $/ Y$ mouse embryonic stem cells. ${ }^{36}$ Flag-DmTrx-2 was immunoprecipitated from lysates with anti-Flag antibodies and immunoblotted with anti-mAIF or anti-Flag antibodies. Lysates are shown as controls. *IgG light chain. (b) Two independent lines carrying the UAS-DmTrx-2-IR transgene were crossed to da-GAL4. Total RNA from third-instar larvae carrying both transgenes was reverse transcribed, and PCR was performed on cDNA using primers specific for DmTrx-2 and G3PDH. da-GAL4 larvae served as control. Control-RT, non-reverse transcribed da-GAL4 total RNA. (c) ey-GAL4,UAS- $\triangle N$-DmAIF/CyO flies were crossed to $w^{1118}$ (control, left panel) or to UAS-DmTrx-2-IR (right panel), and the effect of coexpression of $\triangle N$-DmAIF and DmTrx-2 was evaluated. (d) ey-GAL4,UAS- $\triangle N-D m A I F / C y O$ male flies were crossed to $w^{1118}$ or to two independent lines of UAS$D m T r x-2-I R$ females. Eclosing progenies were tabulated according to whether they carried UAS- $\triangle N$-DmAIF, UAS-DmTrx-2-IR or both transgenes. Note that downregulation of Trx-2 suppresses pupal lethality caused by expression of $\triangle \mathrm{N}-\mathrm{DmAlF}$, thereby increasing the frequency of eclosing flies. Numbers in brackets refer to number of flies counted. For example, in cross 1, $12 \%$ of eclosing progeny received ey-GAL4,UAS- $\triangle N-D m A I F$, indicating that expression of $\triangle N$-DmAIF is semilethal (Mendelian expected frequency is $50 \%$ ). In contrast, in cross $2,45 \%$ of eclosing progeny received both ey-GAL4,UAS- $\triangle N$-DmAIF and UAS-DmTrx-2-IR (expected frequency was also $50 \%$ ), suggesting that downmodulation of DmTrx-2 can significantly suppress $\triangle \mathrm{N}$-DmAIF-induced pupal lethality

representing the full open reading frame. To construct $\triangle \mathrm{N}$-DmAIF (encoding aa 178-674), the primers $5^{\prime}$-TTGCGGCCGCTCCACCATGGCCACGAGTCCGCCC AGTTCTGA-3' (containing an upstream Notl site, Kozak sequence and in-frame ATG, all underlined) and $5^{\prime}$-GCTTCTCTCGGATCCCGTCCATTG-3' were used to amplify the $5^{\prime}$ region of DmAIF by PCR. The PCR product was cloned into the Notl and Clal sites of pBluescript KS II (pBS). Next, LD41427 was digested with Clal and Sall and cloned into pBS to generate the complete $\triangle N$-DmAIF. $\triangle N$-DmAIF was excised using Notl and Xhol and cloned into the pUAST vector. For construction of DmAIF expression constructs for cell transfections, the Myc epitope was tagged to the $\mathrm{N}$ terminus of DmAIF (encoding aa 178-674) or tagged to the $\mathrm{C}$ terminus of fulllength DmAIF using the full-length DmAIF cDNA clone SD03428 as template. Constructs were cloned into pMT (for the full-length DmAIF) or pPAC (for the $\mathrm{N}$-terminal deletion mutant).

Fly crosses. Flies were maintained on standard medium at $25^{\circ} \mathrm{C}$ in a temperature-controlled incubator. The full-length and $\Delta \mathrm{N}$-DmAIF constructs were cloned in the pUAST vector and injected into $w^{1118}$ embryos by standard methods to generate P[UAS-DmAIF-myc] and P[UAS- $\Delta N-D m A I F]$ transformants. At least six independent transgenic lines of P[UAS- $\Delta N-D m A I F]$ were generated, and all showed similar phenotypes when crossed with ey-GAL4 flies. Experiments were carried out with the P[UAS- $\left.\Delta N-D m A I F^{2 A}\right]$ line, in which the transgene is inserted on chromosome 2. A stable line was generated by recombining the ey-GAL4 and
P[UAS- $\triangle N$-DmAIF transgenes and balanced over CyO. This line, denoted ey-GAL4,UAS- $\triangle N-D m A I F$, was used for all experiments, except in Figure $5 \mathrm{~b}$ where a P[UAS- $\triangle N-D m A I F]$ on the third chromosome was used. UAS-DmTrx-2-IR flies (line nos. 36297 and 36298, corresponding to gene CG31884) were obtained from the Vienna Drosophila RNAi Center (Vienna, Austria). UAS-Diap1 and UAS-Diap2 flies were kindly provided by Pascal Meier (Institute of Cancer Research, UK). dark ${ }^{\mathrm{CD} 4}$ represents a strongly hypomorphic mutation in the dark locus; ${ }^{27} \mathrm{dredd}^{\mathrm{B} 118}$ and $p 53^{\text {ns }}$ were obtained from JM Abrams (University of Texas SWMC, Dallas, TX, USA) and are likely null mutants of their respective genes. Two P[UAS-p35] lines (BH1 and BH2) were used and produced similar results. H99/TM6B (no. 1576), P[UAS-p35] (nos. 5072 and 5073), ey-GAL4/CyO (no. 5535), pnr-GAL4/TM3,Ser (no. 3039) and UAS-mitoGFP/CyO lines were obtained from the Bloomington Stock Center. Staging of larvae was based on mouth hook structure and size.

Gene targeting of Drosophila AIF. The donor construct consisted of an $\sim 5$ $\mathrm{kb}$ region spanning the entire DmAIF gene locus (red lines in Figure $2 \mathrm{a}$ ), the last $\sim 200$ amino acids of the neighboring gene BcDNA:LD22320 and the first 21 amino acids of the gene CG15382 (indicated in black in Figure 2a). The region corresponding to nucleotides 257271 and 262278 (AE003584) was PCR amplified from wild-type $w^{1118}$ genomic DNA using primers $5^{\prime}$-CTTCCTGCAGATCAAGTAT GCCTC-3' and 5'-GTCGGAATCTCTTACTGGCGAGG-3'. A 70l-Scel site was cloned into exon 6 (nucleotide 800 of coding region of AY052083). The construct 
was digested with Spel (nucleotide 253), blunted and recircularized, thereby causing a frameshift in exon 3 . Similarly, the construct was digested with $B c h$ (nucleotide 1548), blunted and recircularized, causing a frameshift in exon 6 . The construct was cloned into the Notl site of targeting vector pTV2. ${ }^{33}$

Germline transformants bearing the donor construct were generated. Flies carrying the DmAIF donor element on the $\mathrm{X}$ chromosome were crossed to flies carrying both 7OFLP and 70I-Scel transgenes on the second chromosome. Progenies from this cross were heat shocked during $0-3$ days of development to induce recombinants in the germ line. Eye-color mosaic females of the genotype DmAIF donor; + /7OFLP, 70l-Scel were crossed to ey-FLP males, and progenies were screened for loss of white ${ }^{+}$mosaicism as described. ${ }^{33}$ In the DmAIF mutant characterized in this paper, DmAIF ${ }^{\top 52}$, the $5^{\prime}$ and $3^{\prime}$ variant copies each carry the expected introduced mutations in exons 6 and 3 , respectively. In addition, the $5^{\prime}$ copy carries the exon 3 mutation, which is probably due to a gene conversion event. Such 'extra' mutations frequently occur in gene targeting in flies. ${ }^{33}$ Confirmed $D m A I F^{T 52}$ heterozygous females were crossed to $w^{1118}$ males for $>6$ generations to eliminate potential second-site mutations.

Correct targeting of the DmAIF locus was confirmed by Southern blot of genomic DNA. CDNA probes flanking the targeted DmAIF locus were generated by PCR using primers $5^{\prime}$-GCCTGTTAAGAGCTCTTCAAGAG-3' and $5^{\prime}$-ACAAGAGATTC A GTCAGTTGACC-3' (5' flanking probe); and $5^{\prime}$-ACCACAGTGCTCTGGAACTG GA-3 $3^{\prime}$ and $5^{\prime}$-GAATTGGTTTCAGGCGGTTATCC- $3^{\prime}$ ( $3^{\prime}$ flanking probe). Fly genomic DNA was digested with the indicated restriction enzymes and Southern blotted.

Northern and western blotting, reverse transcription-PCR and in situ hybridizations. Northern blotting of total RNA from flies at various developmental stages was performed using full-length DmAIF as a probe, essentially as described ${ }^{27}$ Whole-mount embryo in situ hybridization assays were conducted with the use of digoxigenin (DIG)-labeled single-stranded antisense and sense DNA probes, as previously described. ${ }^{27}$ Probes were generated by PCR using the full-length DmAIF CDNA clone SD03428 as template and the primers 5'-AGCCAGTGCAAATCACGATGAG-3' and 5'-ATCACGCAGCATGGGCAGGTT $C-3^{\prime}$, comprising the first $\sim 1 \mathrm{~kb}$ of the gene. For reverse transcription-PCR, total RNA was extracted from wandering larvae using TRI reagent (Sigma), treated with DNase I and reverse transcribed using RTG You-Prime-First-Strand Beads (GE Healthcare). PCR was performed in a reaction using combined DmTrx-2 primers (5'-ATGGACAGCTGACCAAGGCATC- $3^{\prime}$ and $5^{\prime}$-CCCACTTAGATATTGGCCTTGA TG-3') and glycerol-3-phosphate dehydrogenase (G3PDH) primers (5'-CCACTGC CGAGGAGGTCAACTA-3' ${ }^{\prime}$ and $5^{\prime}$-GCTCAGGGTGATTGCGTATGCA-3').

Anti-DmAIF antibodies were generated from rabbits immunized with peptides corresponding to DmAIF (aa 661-674), and purified on an affinity column (Pierce). Fly protein lysates were produced by homogenizing larvae in ice-cold RIPA buffer containing protease inhibitors (Roche). Western blotting was performed as previously described. ${ }^{40}$

Immunohistochemistry. Embryos were collected and treated with $\mathrm{AO}$ as described..$^{40}$ Eye discs were dissected from third-instar larvae, stained for $15 \mathrm{~min}$ with $2 \mu \mathrm{g} / \mathrm{ml}$ AO in PBS and imaged immediately. In Supplementary Figure $2 \mathrm{~b}$, wandering larvae were $\gamma$-irradiated with $45 \mathrm{~Gy} ; 5 \mathrm{~h}$ later, eye discs were dissected and stained with $\mathrm{AO}$. For immunostaining, eye discs were fixed in $4 \%$ PFA/PBS for 20 min and washed in PBS containing $0.1 \%$ Triton X-100 (PBS/Triton). Primary antibodies used were as follows: guinea-pig anti-Kr $(1: 600)$, anti-dHb9 antibody (1:500), anti-cleaved caspase 3 (1:50; Cell Signaling) and anti-c-myc $(1 \mu \mathrm{g} / \mathrm{ml}$; Clontech). Antibody labeling was visualized using fluorochrome-conjugated secondary antibodies (1:500; Vector Laboratories or 1:1000; Molecular Probes). Embryos were imaged by confocal microscopy (Leica TCS SP5) and quantified using Image $\mathrm{J}$ software. All cells per segment of the VNC labeled against anti-Kr were quantified, and only the lateral VNC cells peripheral to the horizontal lines labeled against anti- $\mathrm{dH} b 9$ antibody were quantified (yellow brackets in Figure 3).

Assays of mitochondrial function. Drosophila mitochondria were prepared by homogenizing approximately 100 first-instar larvae using a similar procedure to that used for preparing mouse tissue mitochondria, ${ }^{7}$ except that a slightly modified homogenization medium $(250 \mathrm{mM}$ sucrose, $5 \mathrm{mM}$ Tris- $\mathrm{HCl}, 2 \mathrm{mM}$ EGTA, $1 \%(\mathrm{w} / \mathrm{v}) \mathrm{BSA}, \mathrm{pH} 7.4$ at $4^{\circ} \mathrm{C}$ ) was used. Measurement of mitochondrial respiratory activity was performed as described. ${ }^{7}$ For ATP measurements, the luciferase-based ATP bioluminescence kit (Roche) was used. Approximately 50 larvae were homogenized in the lysis buffer provided and centrifuged to remove particulate matter. An aliquot of the supernatant was removed for protein quantitation, and the remainder boiled for $10 \mathrm{~min}$ to destroy endogenous ATPase activity before assessing total ATP levels, according to the manufacturer's instructions.

Acknowledgements. We are grateful to Pascal Meier for the UAS-Diap1 and UAS-Diap2 flies; Georg Dietzl, Kuan-Chung Su, Armen Manoukian and Sam Scanga for help in generating fly transformants; Nazanine Modjtahedi and Barry $\mathrm{J}$ Dickson for helpful discussions; and Teiji Wada Shane Cronin, Tamara Zoranovic, Rubina Yaghubian-Malhami and Cuiping Xia for technical advice. NJ is supported by an NSERC postdoctoral fellowship. GK is supported by grants from the Ligue contre le Cancer (Laboratoire labelisée), Agence Nationale de Recherche, Institut National du Cancer et Cancéropôle lle-de-France. PB and PR are supported by the Integrated European Project Eumitocombat and the Association Française contre les Myopathies. JMP is supported by grants from the Austrian National Bank, IMBA, and the Austrian Ministry of Science.

1. Susin SA, Lorenzo HK, Zamzami N, Marzo I, Snow BE, Brothers GM et al. Molecular characterization of mitochondrial apoptosis-inducing factor. Nature 1999; 397: 441-446.

2. Cande C, Vahsen N, Kouranti I, Schmitt E, Daugas E, Spahr C et al. AlF and cyclophilin A cooperate in apoptosis-associated chromatinolysis. Oncogene 2004; 23: 1514-1521.

3. Klein JA, Longo-Guess CM, Rossmann MP, Seburn KL, Hurd RE, Frankel WN et al. The harlequin mouse mutation downregulates apoptosis-inducing factor. Nature 2002; 419: 367-374.

4. Apostolova N, Cervera AM, Victor VM, Cadenas S, Sanjuan-Pla A, Alvarez-Barrientos A et al. Loss of apoptosis-inducing factor leads to an increase in reactive oxygen species, and an impairment of respiration that can be reversed by antioxidants. Cell Death Differ 2006; 13: 354-357.

5. Brown D, Yu BD, Joza N, Benit P, Meneses J, Firpo M et al. Loss of Aif function causes cell death in the mouse embryo, but the temporal progression of patterning is normal. Proc Natl Acad Sci USA 2006; 103: 9918-9923.

6. Joza N, Oudit GY, Brown D, Benit P, Kassiri Z, Vahsen N et al. Muscle-specific loss of apoptosis-inducing factor leads to mitochondrial dysfunction, skeletal muscle atrophy, and dilated cardiomyopathy. Mol Cell Biol 2005; 25: 10261-10272.

7. Vahsen N, Cande C, Briere JJ, Benit P, Joza N, Larochette $N$ et al. AlF deficiency compromises oxidative phosphorylation. EMBO J 2004; 23: 4679-4689.

8. van Empel VP, Bertrand AT, van der Nagel R, Kostin S, Doevendans PA, Crijns HJ et al. Downregulation of apoptosis-inducing factor in harlequin mutant mice sensitizes the myocardium to oxidative stress-related cell death and pressure overload-induced decompensation. Circ Res 2005; 96: e92-e101.

9. Cheung EC, Melanson-Drapeau L, Cregan SP, Vanderluit JL, Ferguson KL, McIntosh WC et al. Apoptosis-inducing factor is a key factor in neuronal cell death propagated by BAXdependent and BAX-independent mechanisms. J Neurosci 2005; 25: 1324-1334.

10. Culmsee C, Zhu C, Landshamer S, Becattini B, Wagner E, Pellecchia M et al. Apoptosisinducing factor triggered by poly(ADP-ribose) polymerase and Bid mediates neuronal cell death after oxygen-glucose deprivation and focal cerebral ischemia. J Neurosci 2005; 25: 10262-10272.

11. Zhu C, Wang X, Huang Z, Qu L, Xu F, Vahsen $\mathrm{N}$ et al. Apoptosis-inducing factor is a major contributor to neuronal loss induced by neonatal cerebral hypoxia-ischemia. Cell Death Differ 2006; 14: 775-784.

12. Yu SW, Andrabi SA, Wang H, Kim NS, Poirier GG, Dawson TM et al. Apoptosis-inducing factor mediates poly(ADP-ribose) (PAR) polymer-induced cell death. Proc Natl Acad Sci USA 2006; 103: 18314-18319.

13. Wang X, Yang C, Chai J, Shi Y, Xue D. Mechanisms of AlF-mediated apoptotic DNA degradation in Caenorhabditis elegans. Science 2002; 298: 1587-1592.

14. Wissing S, Ludovico P, Herker E, Buttner S, Engelhardt SM, Decker T et al. An AIF orthologue regulates apoptosis in yeast. J Cell Biol 2004; 166: 969-974.

15. Cregan SP, Fortin A, MacLaurin JG, Callaghan SM, Cecconi F, Yu SW et al. Apoptosisinducing factor is involved in the regulation of caspase-independent neuronal cell death. $J$ Cell Biol 2002; 158: 507-517.

16. Susin SA, Daugas E, Ravagnan L, Samejima K, Zamzami N, Loeffler M et al. Two distinct pathways leading to nuclear apoptosis. J Exp Med 2000; 192: 571-580.

17. Danial NN, Korsmeyer SJ. Cell death: critical control points. Cell 2004; 116: 205-219.

18. Dorstyn L, Read S, Cakouros D, Huh JR, Hay BA, Kumar S. The role of cytochrome $c$ in caspase activation in Drosophila melanogaster cells. J Cell Biol 2002; 156: 1089-1098.

19. Means JC, Muro I, Clem RJ. Lack of involvement of mitochondrial factors in caspase activation in a Drosophila cell-free system. Cell Death Differ 2006; 13: 1222-1234.

20. Varkey J, Chen P, Jemmerson R, Abrams JM. Altered cytochrome $c$ display precedes apoptotic cell death in Drosophila. J Cell Biol 1999; 144: 701-710.

21. Zimmermann KC, Ricci JE, Droin NM, Green DR. The role of ARK in stress-induced apoptosis in Drosophila cells. J Cell Biol 2002; 156: 1077-1087.

22. Abdelwahid E, Yokokura T, Krieser RJ, Balasundaram S, Fowle WH, White $K$ Mitochondrial disruption in Drosophila apoptosis. Dev Cell 2007; 12: 793-806. 
23. Goyal G, Fell B, Sarin A, Youle RJ, Sriram V. Role of mitochondrial remodeling in programmed cell death in Drosophila melanogaster. Dev Cell 2007; 12: 807-816.

24. Arama E, Bader M, Srivastava M, Bergmann A, Steller H. The two Drosophila cytochrome C proteins can function in both respiration and caspase activation. EMBO J 2006; 25: 232-243.

25. Hay BA, Wolff T, Rubin GM. Expression of baculovirus P35 prevents cell death in Drosophila. Development 1994; 120: 2121-2129.

26. Kornbluth S, White K. Apoptosis in Drosophila: neither fish nor fowl (nor man, nor worm). J Cell Sci 2005; 118: 1779-1787.

27. Rodriguez A, Oliver H, Zou H, Chen P, Wang X, Abrams JM. Dark is a Drosophila homologue of Apaf-1/CED-4 and functions in an evolutionarily conserved death pathway. Nat Cell Biol 1999; 1: 272-279.

28. Mazzalupo S, Cooley L. Illuminating the role of caspases during Drosophila oogenesis. Cell Death Differ 2006; 13: 1950-1959.

29. Ye H, Cande $\mathrm{C}$, Stephanou NC, Jiang S, Gurbuxani S, Larochette $\mathrm{N}$ et al. DNA binding is required for the apoptogenic action of apoptosis inducing factor. Nat Struct Biol 2002; 9 680-684.

30. Lorenzo HK, Susin SA, Penninger J, Kroemer G. Apoptosis inducing factor (AIF): phylogenetically old, caspase-independent effector of cell death. Cell Death Differ 1999; 6 : 516-524.

31. Mate MJ, Ortiz-Lombardia M, Boitel B, Haouz A, Tello D, Susin SA et al. The crysta structure of the mouse apoptosis-inducing factor AIF. Nat Struct Biol 2002; 9: 442-446.

32. Miramar MD, Costantini P, Ravagnan L, Saraiva LM, Haouzi D, Brothers G et al. NADH oxidase activity of mitochondrial apoptosis-inducing factor. J Biol Chem 2001; 276 16391-16398
33. Rong YS, Titen SW, Xie HB, Golic MM, Bastiani M, Bandyopadhyay P et al. Targeted mutagenesis by homologous recombination in D.melanogaster. Genes Dev 2002; 16 1568-1581.

34. Loeffler M, Daugas E, Susin SA, Zamzami N, Metivier D, Nieminen AL et al. Dominant cell death induction by extramitochondrially targeted apoptosis-inducing factor. FASEB J 2001 15: 758-767.

35. Bauer H, Kanzok SM, Schirmer RH. Thioredoxin-2 but not thioredoxin-1 is a substrate of thioredoxin peroxidase- 1 from Drosophila melanogaster. isolation and characterization of a second thioredoxin in D.melanogaster and evidence for distinct biological functions of Trx-1 and Trx-2. J Biol Chem 2002; 277: 17457-17463.

36. Joza N, Susin SA, Daugas E, Stanford WL, Cho SK, Li CY et al. Essential role of the mitochondrial apoptosis-inducing factor in programmed cell death. Nature 2001; 410: 549-554.

37. Kim JT, Kim KD, Song EY, Lee HG, Kim JW, Kim JW et al. Apoptosis-inducing factor (AIF) inhibits protein synthesis by interacting with the eukaryotic translation initiation factor 3 subunit p44 (elF3g). FEBS Lett 2006; 580: 6375-6383.

38. Wang X, Wang J, Gengyo-Ando K, Gu L, Sun CL, Yang C et al. C.elegans mitochondrial factor WAH-1 promotes phosphatidylserine externalization in apoptotic cells through phospholipid scramblase SCRM-1. Nat Cell Biol 2007; 9: 541-549.

39. Meier P, Silke J, Leevers SJ, Evan GI. The Drosophila caspase DRONC is regulated by DIAP1. EMBO J 2000; 19: 598-611.

40. Chew SK, Akdemir F, Chen P, Lu WJ, Mills K, Daish T et al. The apical caspase dronc governs programmed and unprogrammed cell death in Drosophila. Dev Cell 2004; 7 : 897-907.

\section{Supplementary Information accompanies the paper on Cell Death and Differentiation website (http://www.nature.com/cdd)}

